\title{
Gastrointestinal Symptoms in Autism Spectrum Disorder: A Literature Review
}

\author{
Arlene Mannion • Geraldine Leader
}

Received: 14 September 2013 / Accepted: 24 September 2013 /Published online: 25 October 2013

(C) Springer Science+Business Media New York 2013

\begin{abstract}
The purpose of this literature review was to provide an overview of the research on gastrointestinal (GI) symptoms in autism spectrum disorder. Topics explored include the prevalence of GI symptoms, the importance of studying GI symptoms, the difficulties in diagnosis, as well as the questionnaire measures used to assess gastrointestinal symptoms. Research on the relationships between gastrointestinal symptoms and developmental regression, language and communication, autism severity, challenging behavior, comorbid psychopathology, sleep problems, and sensory issues is also discussed. The use of special diets is discussed also. Finally, recommendations for treatment are given as well as areas where future research is needed.
\end{abstract}

Keywords Autism spectrum disorders · Gastrointestinal symptoms $\cdot$ Comorbidity $\cdot$ Special diets $\cdot$ Treatment $\cdot$ Future research

\section{Introduction}

The most common gastrointestinal (GI) symptoms reported in individuals with autism spectrum disorder (ASD) are chronic constipation, abdominal pain with or without diarrhea, and encopresis as a consequence of constipation (Buie et al. 2010a). Buie et al. (2010a) also commented that other gastrointestinal abnormalities include gastroesophageal reflux disease (GERD), abdominal bloating, disaccharidase deficiencies,

This research was conducted by the first author under the supervision of the second author in partial fulfillment of the requirements for her Ph.D degree in ABA at NUI, Galway.

A. Mannion · G. Leader $(\bowtie)$

Irish Centre for Autism and Neurodevelopmental Research, National

University of Ireland, Galway, Ireland

e-mail: geraldine.leader@nuigalway.ie inflammation of the gastrointestinal tract, and abnormalities of the enteric nervous system.

\section{Gastrointestinal Symptoms and ASD Comorbidity}

Prevalence

The reported prevalence of gastrointestinal problems in children with ASD has ranged from 9 to $91 \%$ (Coury et al. 2012; Ming et al. 2008; Black et al. 2002; Fombonne and Chakrabarti 2001; Ibrahim et al. 2009; Molloy and Manning-Courtney 2003; Mouridsen et al. 2010; Nikolov et al. 2009; Taylor et al. 2002; Valicenti-McDermott et al. 2008; Horvath and Perman 2002; Wang et al. 2011; Parracho et al. 2005; Smith et al. 2009). Wang et al. (2011) commented on the factors that lead to differing rates of GI symptoms across studies. First, there are differences in the target population being studied. Some participants may have different diagnoses on the autism spectrum. Some studies use control groups, while others do not. Second, there are differences in how data is gathered, whether it is by medical records, physicians, or questionnaire-based research. Finally, there are different definitions used for what are considered gastrointestinal symptoms (Wang et al. 2011).

Importance of Studying Gastrointestinal Symptoms

There are many reasons gastrointestinal symptoms in autism need to be researched. First, given that a high prevalence of GI symptoms have been found in the literature, it is possible that GI symptoms affect a large proportion of those with autism. Second, these symptoms may cause pain and discomfort. Individuals that are nonverbal or have little communication skills may not be able to tell parents or caregivers that they are in pain. Third, it is important to recognize that abdominal pain 
or discomfort may act as a setting event for challenging behavior (Buie et al. 2010a). This discomfort and, in turn, challenging behavior can get in the way of the individual acquiring new academic or self-help skills. Mulloy et al. (2010) commented on these biological motivating operations, whereby an upset stomach may act as a motivating operation affecting social consequences. Mulloy et al. (2010) gave the example that a child with an upset stomach may find academic work more demanding than if they did not have an illness. Therefore, they may engage in increasing levels of challenging behavior to escape the demand. Finally, an individual's quality of life can be affected if they present with GI symptoms. Williams et al. (2010) found that children with ASD with GI symptoms had lower quality of life compared to children without GI symptoms. GI complaints are therefore associated with overall decreased health-related quality of life

\section{Difficulty of Diagnosis}

GI symptoms are difficult to diagnose in individuals with ASD for several reasons. First, clinical practice guidelines in place for the diagnosis of ASD do not include routine consideration of potential gastrointestinal or other medical conditions (Buie et al. 2010a). Second, many individuals with ASD are nonverbal or have communication difficulties. Because of this, they may not be able to express pain or discomfort in a typical manner. They may not be able to communicate their symptoms as clearly as those who are typically developing. Those who can verbally communicate may have difficulty describing subjective experiences or symptoms (Buie et al. 2010a). Third, those with ASD may present with GI symptoms in atypical ways. One may assume that if an individual has abdominal or other discomfort that they would touch their abdomen. However, this is not necessarily the case for those with ASD. Gastrointestinal disorders can present as nongastrointestinal problems (Buie et al. 2010a). For example, individuals may present with sleep problems or challenging behavior. Where they present with vocal stereotypy or repetitive behavior, it could be incorrectly attributed to being symptoms of autism. Fourth, diet may play a role in GI symptoms. Kuddo and Nelson (2003) commented that the insistence on sameness in autism may lead children to demand stereotyped diets which are lacking in fibers, fluids, or other constitutes. Finally, if children are on medication, this can have side effects. Kuddo and Nelson (2003) comment that most medication administered to children with autism can influence gut function.

\section{Measures Used to Assess Gastrointestinal Symptoms}

While some studies used medical history to assess gastrointestinal symptoms (Molloy and Manning-Courtney 2003; Nikolov et al. 2009; Maenner et al. 2012), a variety of questionnaires have also been used. As well as past medical history, Nikolov et al. (2009) used the Side Effects Review Form (Research Units on Pediatric Psychopharmacology). The form was designed to establish whether certain problems are present prior to drug exposure and to track changes. In the study by Nikolov et al. (2009), a pretreatment rating of moderate or severe on the Side Effects Review Form in response to one or more GI questions was classified as the presence of a GI symptom. Hansen et al. (2008) used the CHARGE (Childhood Autism Risks from Genetics and the Environment) gastrointestinal history form, which includes 10 items describing current gastrointestinal symptoms as well as questions relating to food allergies and diet restrictions.

Valicenti-McDermott et al. (2008) developed The Gastrointestinal Questionnaire, derived from the Clinical Diagnostic Questionnaire for Pediatric Functional Gastrointestinal Disorders, as developed by the Committee on Childhood Functional Gastrointestinal Disorders Multinational Working Teams to Develop Criteria for Functional Disorders (Rome II). The Gastrointestinal Questionnaire includes questions on current GI symptoms, as well as lifetime gastrointestinal or feeding problems. Gorrindo et al. (2012) used the Questionnaire on Pediatric Gastrointestinal Symptoms-Rome III Version (QPGS) which is a 71item parent report instrument that assesses GI symptoms and classifies functional GI disorders according to Rome III criteria. It is available online at http://romecriteria.org/. Gorrindo et al. (2012) also included clinical evaluation in their study. The authors found that parent report of any gastrointestinal dysfunction in those with ASD was highly concurrent $(92.1 \%)$ with a clinical diagnosis of any gastrointestinal dysfunction.

Chandler et al. (2013) constructed a 20-item GI symptom questionnaire. Questions were asked by current (last 3 months) and past (prior to the last 3 months symptoms). The GI symptoms included persistent vomiting; stool consistency; abdominal pain; abdominal pain associated with food, bowel movement, or sleep; constipation; subjective difficulties with bowel movements, stool withholding, and soiling; diarrhea, weight loss, mouth ulcers, and presence of mucus or blood in the stools.

The Gastrointestinal Symptom Inventory (Autism Treatment Network 2005) is a 35-item questionnaire that was developed by the Autism Treatment Network (ATN). The ATN is the first network of hospitals and physicians dedicated to developing a model of comprehensive medical care for children and adolescents with autism through $17 \%$ participating institutions in the USA and Canada. In the inventory, there are additional items should a participant exhibit certain symptomatology, and therefore includes 77 items in total. This tool has not been validated. It was based on previous questionnaires and on clinical symptom assessment for children with autism and identified gastrointestinal disorders. It measures questions about the presence and duration of GI symptoms. The inventory is scored initially dichotomously, i.e., whether or not the child has any of the gastrointestinal 
symptoms. The GI symptoms it measures are abdominal pain, nausea, bloating, diarrhea, or other GI symptom. The inventory also allows branching into specific areas of symptomatology: abdominal pain, abnormal bowel movements, reflux, and food insensitivity. These branches will allow determination of rates of these categories as well.

Mazurek et al. (2013b) used the GI Symptom Inventory in their research. It was also used in Williams et al. (2010, 2012a, b) and Mannion et al. (2013). The GI Symptom Inventory is no longer used by the ATN as part of its registry battery. Instead, questions are included about gastrointestinal symptoms in the ATN Parent Baseline Assessment. The following symptoms are assessed: nausea/vomiting, reflux, diarrhea, constipation, and stomach/abdominal pain. Mazurek et al. (2013a) used the Parent Baseline Assessment in their research.

\section{Regression}

Valicenti-McDermott et al. (2008) found that children with autism spectrum disorder who presented with language regression had more gastrointestinal problems $(84 \%)$ than those without language regression (61\%). The authors also found that abnormal stool patterns were reported more frequently in the group with language regression (42 vs. $12 \%$ ). Niehus and Lord (2006) found that the medical records of children with ASD and regression indicated significantly more parental reports of bloody stools than those with ASD and no regression. Though nonsignificant, those with ASD and regression had more chronic diarrhea and stool complaints than those with ASD and no regression or those that were typically developing.

Baird et al. (2008) investigated factors associated with regression in children with autism. Current gastrointestinal symptoms varied across the three groups in the study: those with no regression, lower level regression, and definite language regression. Current gastrointestinal symptoms varied across regression groups, but the rate was higher in the no regression group than the lower or definite regression groups. In terms of past gastrointestinal symptoms, there was no group difference found between those with no regression, lower, or definite regression. This is supported by Chandler et al. (2013) who found that there were no differences between ASD children with and without a history of regression for current and past GI symptoms. Similarly, Molloy and Manning-Courtney (2003) found that developmental regression was not significantly associated with gastrointestinal symptoms. Hansen et al. (2008) found no significant differences between the children with ASD with or without regression in terms of gastrointestinal symptoms.

\section{Language and Communication}

Gorrindo et al. (2012) examined expressive language and social responsiveness as communication variables in their study. They found that children with ASD and GI symptoms showed higher levels of social impairment than those with ASD only. However, this social impairment was not associated with impaired language, as there was no difference in scores of those with ASD and GI symptoms that were verbal and nonverbal. Gorrindo et al. (2012) also investigated the risk factors for constipation as constipation was found to be the most common GI symptom in their study. The authors found that younger, more socially impaired and nonverbal children had increased odds for constipation. In contrast, Chandler et al. (2013) did not find a difference between verbal ability in children with and without reported pain abdominal pain or constipation. This is supported by Williams et al. (2012b) who found that rates of GI complaints did not differ between four verbal ability groups: nonverbal, some words, phrase speech, and verbal.

\section{Autism Severity}

Wang et al. (2011) classified autism severity into three groups: full autism, almost autism, and not quite autism. Increased autism symptom severity was associated with increased odds of having significantly more GI problems being reported. Specifically, having full autism or almost autism was most highly associated with experiencing GI problems (Wang et al. 2011). However, Molloy and Manning-Courtney (2003) found that frequency of gastrointestinal symptoms did not vary by age, gender, race, or severity of autism. In support of this finding, Nikolov et al. (2009) also found that those with gastrointestinal problems were no different from those without gastrointestinal problems in autism symptom severity, demographic characteristics, or measures of adaptive functioning. Chandler et al. (2013) found that there was no significant association between autism severity and current and past GI symptoms in the ASD group. Williams et al. (2010) found that presence of GI problems did not differ by gender, ASD subtype, race, or IQ. However, Williams et al. (2012b) found that chronic GI symptoms were more likely in children with Asperger's than autism.

\section{Challenging Behavior}

Horvath et al. (1999) commented that unrecognized gastrointestinal symptoms and disorders may contribute to the behavioral problems of nonverbal children with autism, such as sudden irritability and aggressive behavior. Mazurek et al. (2013a) investigated the relationship between aggression and other variables, including gastrointestinal symptoms in children with ASD. Children with aggression had significantly greater difficulties with GI problems than those without aggression. However, GI problems did not emerge as significant predictors of aggression. 
Children with GI problems were more likely to present with argumentative, oppositional, or destructive behaviors than those without GI problems (Maenner et al. 2012). Tantrum behaviors were more common in those with GI problems than those without, but the association did not reach statistical significance. No association was found between presence of GI problems and stereotypic/repetitive behaviors or selfinjurious behaviors (Maenner et al. 2012).

\section{Comorbid Psychopathology}

Williams et al. (2010) found that children aged 1-5 years with GI symptoms had higher total scores on the Child Behavior Checklist (CBCL; Achenbach and Rescorla 2000) and for the emotionally reactive, anxious/depressed, somatic complaints, internalizing problems, affective problems, and anxiety problems subscales. The authors also found that children aged 618 years had higher total scores on the CBCL (Achenbach and Rescorla 2001) and on all subscales. Maenner et al. (2012) found that mood disturbances were more common in children with GI problems than those without but this association did not reach statistical significance. In terms of other comorbid disorders, children with ASD and GI problems were significantly more likely than those without GI problems to have cooccurring cerebral palsy- and seizure-like activity (Maenner et al. 2012).

Mannion and Leader (2013) investigated predictors of GI symptoms in children with ASD. In the study, the total number of GI symptoms predicted total scores on the Autism Spectrum Disorder-Comorbid for Children (ASD-CC) (Matson and González 2007). The ASD-CC is a questionnaire used to assess comorbid psychopathology in those with autism. Specifically, abdominal pain and constipation also predicted conduct behavior. Diarrhea predicted tantrum behavior. Nausea predicted worry/depressed behavior, avoidant behavior, and conduct behavior. The worry/depressed subscale and the avoidant behavior subscale of the ASD-CC form a measure of anxiety (Davis et al. 2011).

Other authors have also investigated the relationship between GI problems and anxiety. Nikolov et al. (2009) found that when compared to children without gastrointestinal problems, those with gastrointestinal problems showed greater symptom severity on measures of irritability, anxiety, and social withdrawal. Williams et al. (2012b) found that clinical anxiety is associated with chronic GI symptoms in children with autism. Chronic GI complaints were greater in children with clinical anxiety compared to those with no anxiety. Mazurek et al. (2013b) found that children with chronic constipation, chronic diarrhea, chronic bloating, chronic nausea, and chronic abdominal pain had higher anxiety than those without these symptoms. Those with more chronic GI problems had higher anxiety scores than those with no chronic GI problems. Anxiety contributed to the prediction of chronic constipation, chronic bloating, chronic nausea, and chronic abdominal pain, but not to the prediction of chronic diarrhea.

\section{Sleep Problems}

Horvath and Perman (2002) commented that unrecognized gastrointestinal symptoms may lead to night time awakenings in nonverbal children with autism. Maenner et al. (2012) found that children with sleep abnormalities were more likely to have a medically documented history of GI problems, than those without. Williams et al. (2010) found that sleep problems occurred most frequently in those with gastrointestinal problems $(50 \%)$ than those without $(37 \%)$. Williams et al. (2012a) found that $24.5 \%$ of children with autism had sleep problems and chronic GI symptoms, while $25.2 \%$ had neither sleep nor GI problems. It was found that $42.5 \%$ had sleep problems only, while $7.8 \%$ had a chronic GI complaint only. Sleep problems occurred in $84 \%$ of children with chronic nausea, $82 \%$ of children with chronic diarrhea, $81 \%$ of children with chronic bloating, $79 \%$ of children with chronic constipation, and $78 \%$ of children with chronic abdominal pain.

Mannion et al. (2013) investigated predictors of sleep problems in children with ASD. The total number of GI symptoms predicted higher rates of sleep problems. Undereating, avoidant behavior, and gastrointestinal symptoms predicted sleep problems. Specifically, abdominal pain predicted sleep anxiety. Undereating, avoidant behavior, and the five GI symptoms (constipation, diarrhea, nausea, abdominal pain, and bloating) predicted parasomnias and daytime sleepiness. Mannion and Leader (2013) found that sleep problems predicted gastrointestinal symptoms. Specifically, sleep disordered breathing and daytime sleepiness predicted both abdominal pain and bloating. Sleep anxiety predicted abdominal pain. It was found that $67.8 \%$ of individuals had both sleep problems and gastrointestinal symptoms, while only $8 \%$ had neither sleep problems nor gastrointestinal symptoms. Sleep problems occurred in $92.3 \%$ of those with nausea and in $91.1 \%$ of those with abdominal pain. Sleep problems occurred in $90.9 \%$ of those with bloating. Sleep problems occurred in $90 \%$ of those with diarrhea and $83.7 \%$ of those with constipation. It was found that $11.5 \%$ had gastrointestinal symptoms only, while $12.6 \%$ had sleep problems only.

\section{Sensory Issues}

Mazurek et al. (2013b) investigated the relationship between sensory over-responsivity and GI problems. They found that those with chronic GI problems had higher levels of sensory over-responsivity. Increasing numbers of GI problems were associated with higher levels of sensory over-responsivity. Sensory over-responsivity also predicted chronic GI problems. 


\section{Special Diets}

Perrin et al. (2012) investigated complementary and alternative medicine (CAM) use in children with autism. Parents of children with autism were asked if their children use any of the following treatments: acupuncture, chelation, chiropractic, or hyperbaric oxygen therapy; dietary supplements (vitamin supplements, probiotics, antifungal agents, digestive enzymes, glutathione, sulfation, amino acids, or essential fatty acids); and special diets(classified as gluten free, casein free, Feingold, no processed sugars, no salicylates, or other). Special diets usage versus no CAM usage was investigated in the study also. Parents reported higher rates of CAM usage in general and for special diets when they also reported GI problems. The authors commented that families seek to address problematic behaviors or symptoms using CAM and that parents whose children have GI symptoms may try both dietary changes and other CAM treatments to improve their child's nutrition and symptoms.

Pennesi and Klein (2012) investigated the effectiveness of the gluten-free, casein-free (GFCF) diet in children with ASD. The authors found the diet to be effective in improving ASD behaviors, physiological behaviors, and social behaviors for children with GI symptoms (specifically, constipation and diarrhea) compared to children with no GI symptoms. The authors suggested that children predisposed to GI abnormalities might particularly benefit more from a GFCF dietary intervention. However, the authors also commented that the findings reported may be highly sensitive to parental perceptions and that the high effectiveness ratings may be explained by a placebo effect. In terms of future research, the authors stated that the priority of future research should be to define the immunological and GI diagnoses and symptoms that best predict those individuals who will be most responsive to the gluten-free, casein-free diet.

Mulloy et al. (2010) conducted a systematic review on gluten-free and casein-free diets in the treatment of autism spectrum disorder. The authors concluded that the published studies they located do not support the use of GFCF diets in the treatment of ASD. The authors commented on negative consequences for the use of the GFCF diet, such as use of treatment resources, stigmatization, and reduced cortical bone thickness. The authors recommended that should a child with ASD experience behavioral changes seemingly associated with change in diet, practitioners should consider testing the child for allergies and food intolerances and eliminate identified allergens or irritants from their environment.

\section{Recommendations for Treatment}

Buie et al. (2010b) commented that children with ASD can benefit from the adaptation of general pediatric guidelines for diagnosing GI symptoms. The authors provided a review of guidelines to diagnose and treat GI symptoms using current general pediatric guidelines, until specific guidelines are designed for those with ASD. Information on differential diagnosis, evaluation, and treatment considerations are provided for chronic abdominal pain, constipation, chronic diarrhea, and GERD in children with ASD.

Furuta et al. (2012) developed an algorithm to help healthcare providers identify, evaluate and manage constipation in children with ASD. The consensus among the authors is that (1) subtle or atypical symptoms might indicate the presence of constipation; (2) screening, identification, and treatment through a deliberate approach for underlying causes of constipation is appropriate; (3) diagnostic-therapeutic intervention can be provided when constipation is documented; and (4) careful follow-up after any intervention be performed to evaluate effectiveness and tolerance of the therapy (Furuta et al. 2012). According to the algorithm, any child with atypical behaviors should be evaluated for constipation. Examples of these behaviors include self-injurious behavior, posturing, grimacing, holding the abdomen, squeezing the legs together, or walking around with a narrow gait to hold the stool in (Furuta et al. 2012).

Buie et al. (2010a) commented that integrating behavioral and biomedical roles can be advantageous. Firstly, functional behavioral assessments can be used in order to interpret the function of challenging behavior. Second, it is important to be aware of pain and discomfort can function as a setting event for challenging behavior. Third, functional communication skills may be taught. Buie et al. (2010a) commented that it would be useful for diagnosis to teach a child to identify the location and type of pain they are experiencing. Finally, the individual could be taught skills for coping with a task demand appropriately during moments of pain or discomfort (Buie et al. 2010a).

\section{Future Research}

Coury et al. (2012) developed a research agenda for gastrointestinal conditions. They defined four priority areas for research: epidemiology of GI conditions in ASD, underlying pathology, treatment and outcome, and nutrition. There is a need for rigorously designed prevalence studies in order to identify risk factors including clinical and behavioral indicators of GI problems, identify atypical presentations of GI disorders in ASD, and identify subpopulations within ASD that have GI symptoms (Coury et al., 2012).

As evidenced by the lack of research on adults with autism in the current review, there is a real need for more research to be conducted with adults with autism to investigate the relationship between GI problems and autism in adults. Edwards et al. (2012) found that in intervention research on autism, 
only $1.7 \%$ of participants were 20 years or more. In autism research, in general, there is a need for adult research and this is especially so in relation to gastrointestinal symptoms.

\section{References}

Achenbach, T. M., \& Rescorla, L. A. (2000). Manual for the ASEBA preschool forms and profiles. Burlington, VT: University of Vermont Department of Psychiatry.

Achenbach, T. M., \& Rescorla, L. A. (2001). Manual for the ASEBA school-age forms and profiles. Burlington, VT: University of Vermont, Research Center for Children, Youth, and Families.

Autism Treatment Network. (2005). GI Symptom Inventory Questionnaire, vers. 3.0. New York, NY: Autism Speaks.

Baird, G., Charman, T., Pickles, A., Chandler, S., Loucas, T., Meldrum, D., Carcani-Rathwell, I., Serkana, D., \& Simonoff, E. (2008). Regression, developmental trajectory and associated problems in disorders in the autism spectrum: the SNAP study. Journal of Autism and Developmental Disorders, 38, 1827-1836. doi:10.1007/ s10803-008-0571-9.

Black, C., Kaye, J. A., \& Jick, H. (2002). Relation of childhood gastrointestinal disorders to autism: nested case-control study using data from the UK general practice database. British Medical Journal, 325, 419-421. doi:10.1136/bmj.325.7361.419.

Buie, T., Campbell, D. B., Fuchs, G. J., III, Furuta, G. T., Levy, J., VandeWater, J., Whitaker, A. H., Atkins, D., Bauman, M. L., Beaudet, A. L., Carr, E. G., Gershon, M. D., Hyman, S. L., Jarapinyo, P., Jyonouchi, H., Kooros, K., Kushak, R., Levitt, P., Levy, S. E., Lewis, J. D., Murray, K. F., Natowicz, M. R., Sabra, A., Wershil, B. K., Weston, S. C., Zeltzer, L., \& Winter, H. (2010a). Evaluation, diagnosis, and treatment of gastrointestinal disorders in individuals with ASDs: a consensus report. Pediatrics, 125, S1S18. doi:10.1542/peds.2009-1878C.

Buie, T., Fuchs, G. J., III, Furuta, G. T., Kooros, K., Levy, J., Lewis, J. D., Wershil, B. K., \& Winter, H. (2010b). Recommendations for evaluation and treatment of common gastrointestinal problems in children with ASDs. Pediatrics, 124, S19-S29. doi:10.1542/peds.20091878D.

Chandler, S., Carcani-Rathwell, I., Charman, T., Pickles, A., Loucas, T., Meldrum, D., Simonoff, E., Sullivan, P., \& Baird, G. (2013). Parentreported gastro-intestinal symptoms in children with autism spectrum disorders. Journal of Autism and Developmental Disorders. doi:10.1007/s10803-013-1768-0.

Coury, D. L., Ashwood, P., Fasano, A., Fuchs, G., Geraghty, M., Kaul, A., Mawe, G., Patterson, P., \& Jones, N. (2012). Gastrointestinal conditions in children with autism spectrum disorder: developing a research agenda. Pediatrics. doi:10.1542/peds.2012-0900N.

Davis, T. E., Moree, B. N., Dempsey, T., Reuther, E. T., Fodstad, J. C., Hess, J. A., Jenkins, W. S., \& Matson, J. L. (2011). The relationship between autism spectrum disorders and anxiety: the moderating effect of communication. Research in Autism Spectrum Disorders, 5, 324-329. doi:10.1016/j.rasd.2010.12.002.

Edwards, T. L., Watkins, E. E., Lotfizadeh, A. D., \& Poling, A. (2012). Intervention research to benefit people with autism: how old are the participants? Research in Autism Spectrum Disorders, 6, 996-999. doi:10.1016/j.rasd.2011.11.002.

Fombonne, E., \& Chakrabarti, S. (2001). No evidence for a new variant of measles-mumps-rubella-induced autism. Pediatrics, 108(4), e58. doi:10.1542/peds.108.4.e58.

Furuta, G. T., Williams, K., Kooros, K., Kaul, A., Panzer, R., Coury, D. L., \& Fuchs, G. (2012). Management of constipation in children and adolescents with autism spectrum disorders. Pediatrics, 130 (Suppl. 2), S98-S105. doi:10.1542/peds.2012-0900H.
Gorrindo, P., Williams, K. C., Lee, E. B., Walker, L. S., McGrew, S. G., \& Levitt, P. (2012). Gastrointestinal dysfunction in autism: parental report, clinical evaluation, and associated factors. Autism Research, 5(2), 101-108. doi:10.1002/aur.237.

Hansen, R. L., Ozonoff, S., Krakowiak, P., Angkustsiri, K., Jones, C., Deprey, L. J., Le, D., Croen, L. A., \& Hertz-Picciotto, I. (2008). Regression in autism: prevalence and associated factors in the CHARGE study. Ambulatory Pediatrics, 8(1), 25-31. doi:10.1016/j.ambp.2007.08.006.

Horvath, K., Papadimitriou, J. C., Rabsztyn, A., Drachenberg, C., \& Tyson Tildon, J. (1999). Gastrointestinal abnormalities in children with autistic disorder. The Journal of Pediatrics, 135(5), 559-563.

Horvath, K., \& Perman, J. A. (2002). Autistic disorder and gastrointestinal disease. Current Opinion in Pediatrics, 14, 583-587. doi:10. 1097/00008480-200210000-00004.

Ibrahim, S. H., Voigt, R. G., Katusic, S. K., Weaver, A. L., \& Barbaresi, J. (2009). Incidence of gastrointestinal symptoms in children with autism: a population based study. Pediatrics, 124, 680-686. doi: 10.1542/peds.2008-2933.

Kuddo, T., \& Nelson, K. B. (2003). How common are gastrointestinal disorders in children with autism? Current Opinion in Pediatrics, 15, 339-343. doi:10.1097/00008480-200306000-00020.

Maenner, M. J., Arneson, C. L., Levy, S. E., Kirby, R. S., Nicholas, J. S., \& Durkin, M. S. (2012). Brief report: association between behavioral features and gastrointestinal problems among children with autism spectrum disorder. Journal of Autism and Developmental Disorders, 42, 1520-1525. doi:10.1007/s10803-011-1379-6.

Mannion, A. \& Leader, G. (2013). An analysis of the predictors of comorbid psychopathology, gastrointestinal symptoms and epilepsy in children and adolescents with autism spectrum disorder. Research in Autism Spectrum Disorders (in press).

Mannion, A., Leader, G., \& Healy, O. (2013). An investigation of comorbid psychological disorders, sleep problems, gastrointestinal symptoms and epilepsy in children and adolescents with autism spectrum disorder. Research in Autism Spectrum Disorders, 7, 3542. doi:10.1016/j.rasd.2012.05.002.

Matson, J. L., \& González, M. L. (2007). Autism spectrum disorderscomorbidity-child version. Baton Rouge, LA: Disability Consultants, LLC.

Mazurek, M. O., Kanne, S. M., \& Wodka, E. L. (2013a). Physical aggression in children and adolescents with autism spectrum disorders. Research in Autism Spectrum Disorders, 7, 455-465. doi:10. 1016/j.rasd.2012.11.004.

Mazurek, M. O., Vasa, R. A., Kalb, L. G., Kanne, S. M., Rosenberg, D., Keefer, A., Murray, D. S., Freedman, B., \& Lowery, L. A. (2013b). Anxiety, sensory over-responsivity, and gastrointestinal problems in children with autism spectrum disorders. Journal of Abnormal Child Psychology, 41, 165-176. doi:10.1007/s10802-012-9668-x.

Ming, X., Brimacombe, M., Chaaban, J., Zimmerman-Bier, B., \& Wagner, G. C. (2008). Autism spectrum disorders: concurrent clinical disorders. Journal of Child Neurology, 23, 6-13.

Molloy, C. A., \& Manning-Courtney. (2003). Prevalence of chronic gastrointestinal symptoms in children with autism and autistic spectrum disorders. Autism, 7, 165-171. doi:10.1177/1362361303007002004.

Mouridsen, S. E., Rich, B., \& Isager, T. (2010). A longitudinal study of gastrointestinal diseases in individuals diagnosed with infantile autism as children. Child Care Health and Development, 36(3), 437443. doi:10.1111/j.1365-2214.2009.01021.x.

Mulloy, A., Lang, R., O’Reilly, M., Sigafoos, J., Lancioni, G., \& Rispoli, M. (2010). Gluten-free and casein-free diets in the treatment of autism spectrum disorders: a systematic review. Research in Autism Spectrum Disorders, 4, 328-339. doi:10.1016/j.rasd.2009.10.008.

Niehus, R., \& Lord, C. (2006). Early medical history of children with autism spectrum disorders. Developmental and Behavioral Pediatrics, 27(2), S120-S127. doi:10.1097/00004703-200604002-00010.

Nikolov, R. N., Bearss, K. E., Lettinga, J., Erickson, C., Rodowski, M., Aman, M. G., McCracken, J. T., McDougle, J., Tierney, E., Vitello, 
B., Arnold, L. E., Shan, B., Posey, D. J., Ritz, L., \& Scahill, L. (2009). Gastrointestinal symptoms in a sample of children with pervasive developmental disorders. Journal of Autism and Developmental Disorders, 30, 405-413. doi:10.1007/s10803-008-0637-8.

Parracho, H. M. R. T., Bingham, M. O., Gibson, G. R., \& McCartney, A. L. (2005). Differences between the gut microflora of children with autistic spectrum disorders and that of healthy children. Journal of Medical Microbiology, 54(10), 987-991. doi:10.1099/jmm.0. 46101-0.

Pennesi, C. M., \& Klein, L. C. (2012). Effectiveness of the gluten-free, casein-free diet for children diagnosed with autism spectrum disorder: based on parental report. Nutritional Neuroscience, 15(2), 8591. doi:10.1179/1476830512Y.0000000003.

Perrin, J. M., Coury, D. L., Hyman, S. L., Cole, L., Reynolds, A. M., \& Clemons, T. (2012). Complementary and alternative medicine use in a large pediatric autism sample. Pediatrics, 130 (Suppl. 2), S77-S82. doi:10.1542/peds.2012-0900E.

Smith, R. A., Farnworth, H., Wright, B., \& Allgar, V. (2009). Are there more bowel symptoms in children with autism compared to normal children and children with other developmental and neurological disorders? A case control study. Autism, 13(4), 343-355. doi:10. 1177/1362361309106418.

Taylor, B., Miller, E., Lingam, R., Andrews, N., Simmons, A., \& Stowe, J. (2002). Measles, mumps, and rubella vaccination and bowel problems or developmental regression in children with autism: population study. British Medical Journal, 324, 393-396. doi:10. 1136/bmj.324.7334.393.

Valicenti-McDermott, M. D., McVicar, K., Cohen, H. J., Wershil, B. K., \& Shinnar, S. (2008). Gastrointestinal symptoms in children with an autism spectrum disorder and language regression. Pediatric Neurology, 39(6), 392-398. doi:10.1016/j.pediatrneurol.2008.07.019.

Wang, L. W., Tancredi, D. J., \& Thomas, D. W. (2011). The prevalence of gastrointestinal problems in children across the United States with autism spectrum disorders from families with multiple affected members. Journal of Developmental and Behavioral Pediatrics, 32, 351-360. doi:10.1097/DBP/0b013e31821bd06a.

Williams, K. C., Fuchs, G. J., Furuta, G. T., Marcon, M. A., \& Coury, D. L. (2010). Clinical features associated with GI symptoms in autism spectrum disorders (ASD). Gastroenterology. doi:10.1016/S00165085(10)60337-X.

Williams, K. C., Christofi, F. L., Clemmons, T., Rosenberg, D., \& Fuchs, G. J. (2012a). Association of chronic gastrointestinal symptoms with sleep problems may help identify distinct subgroups of autism spectrum disorders. Gastroenterology. doi:10.1016/S0016-5085(12)62767-X.

Williams, K. C., Christofi, F. L., Clemmons, T., Rosenberg, D., \& Fuchs, G. J. (2012b). Chronic GI symptoms in children with autism spectrum disorders are associated with clinical anxiety. Gastroenterology. doi:10.1016/S0016-5085(12)60303-5. 